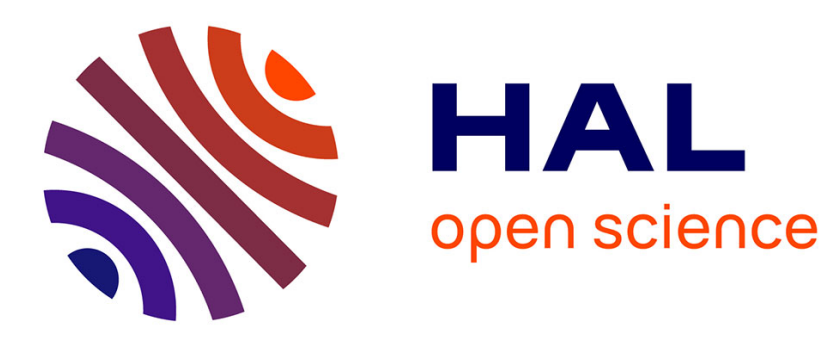

\title{
Effet antalgique aigu du paracétamol en soins primaires : des preuves incomplètes
}

Marion Gougain, Moreau Alain, Rémy Boussageon, Gisèle Pickering, François

Gueyffier

\section{- To cite this version:}

Marion Gougain, Moreau Alain, Rémy Boussageon, Gisèle Pickering, François Gueyffier. Effet antalgique aigu du paracétamol en soins primaires: des preuves incomplètes. Thérapie, 2017, 72 (5), pp.609 - 613. 10.1016/j.therap.2017.02.004 . halshs-01866986

\section{HAL Id: halshs-01866986 \\ https://shs.hal.science/halshs-01866986}

Submitted on 3 Sep 2018

HAL is a multi-disciplinary open access archive for the deposit and dissemination of scientific research documents, whether they are published or not. The documents may come from teaching and research institutions in France or abroad, or from public or private research centers.
L'archive ouverte pluridisciplinaire HAL, est destinée au dépôt et à la diffusion de documents scientifiques de niveau recherche, publiés ou non, émanant des établissements d'enseignement et de recherche français ou étrangers, des laboratoires publics ou privés. 
COMMUNICATION RAPIDE

Titre : Effet antalgique aigu du paracétamol en soins primaires : des preuves incomplètes

Titre courant : Paracétamol en soins primaires

Titre anglais : Acute analgesic effect of paracetamol in primary care : incomplete evidence

Marion Gougain ${ }^{\mathrm{a}}$, Alain Moreau ${ }^{\mathrm{b}}$, Rémy Boussageon ${ }^{\mathrm{c}}$, Gisèle Pickering ${ }^{\mathrm{d}, \mathrm{e}}$, François

Gueyffier ${ }^{\mathrm{a}, \mathrm{f}}$

${ }^{\text {a } U M R ~ 5558, ~ U C B L ~ L y o n ~ 1, ~} 69008$ Lyon, France

${ }^{\mathrm{b}}$ Collège Universitaire de Médecine Générale, UCBL Lyon 1, 69003 Lyon, France

${ }^{\mathrm{c}}$ Département de Médecine Générale, Université de Poitiers, 86073 Poitiers, France

d CHU Clermont-Ferrand, Inserm CIC 1405, Centre de Pharmacologie Clinique, F-63003 Clermont-Ferrand, France.

${ }^{\text {e }}$ Clermont Université, Pharmacologie Fondamentale et Clinique de la Douleur, Inserm U1107 Neuro-Dol, F-63000 Clermont-Ferrand, France

${ }^{\mathrm{f}}$ Service de Pharmacologie Toxicologie, Hospices Civils de Lyon, France

Auteur correspondant : Marion Gougain, Service de pharmacologie clinique et toxicologie, UMR 5558, faculté RTH Laennec, 8 rue Guillaume Paradin, 69376 Lyon Cedex 08, France

E-mail : marion.gougain@ gmail.com

\section{Résumé en français :}

Objectif : Evaluer l'effet antalgique d'une prise orale de paracétamol versus placebo chez l'adulte, en soins primaires.

Méthodes : Méta-analyse réalisée à partir des bases MEDLINE, CENTRAL et EMBASE, incluant tous les essais randomisés en parallèle versus placebo évaluant l'effet antalgique d'une prise orale unique de paracétamol dans les 6 heures suivant la prise.

Résultats : Un auteur a sélectionné les articles et extrait les données. Cinq études concernaient les maux de gorge (842 patients), 12 les céphalées (3433 patients), aucune n'était exploitable pour les lombalgies. Quel que soit le critère de jugement considéré, le paracétamol était 
supérieur au placebo (Nombre de patients Nécessaire à Traiter $(\mathrm{NNT})=4-5$; Différence de Moyenne Standardisée $(\mathrm{DMS})=0.51-0.92)$.

Conclusion: Des études de soins primaires restent nécessaires pour valider l'efficacité antalgique du paracétamol dans l'ensemble des situations courantes.

Mots-clés : antalgique, paracétamol, placebo, soins primaires, méta-analyse.

\section{Résumé en anglais :}

This meta-analysis assessed the analgesic effect of a single oral dose of paracetamol versus placebo in adults, in primary care. Parallel randomized studies were searched on MEDLINE, CENTRAL, and EMBASE. We found five studies on sore throat (842 patients) and 12 studies on headache (3433 patients). None related to low back pain. Regardless of the outcome, paracetamol was superior to placebo (Number Need to Treat $(\mathrm{NNT})=4-5$; Standardized Mean Difference $(\mathrm{SMD})=0.51-0.92)$. Additional clinical trials in other primary care conditions must be conducted.

Keywords : analgesic, acetaminophen, placebo, primary health care, meta-analysis.

Abréviations :

\begin{tabular}{|l|l|}
\hline ANSM & Agence Nationale de Sécurité du Médicament et des produits de santé \\
\hline COX & Cyclo-oxygénases \\
\hline DMS & Différence de Moyenne Standardisée \\
\hline DR & Différence de Risque \\
\hline ECR & Essai Clinique Randomisé \\
\hline h & Heures \\
\hline IC95\% & Intervalle de Confiance à 95\% \\
\hline mg & Milligrammes \\
\hline NNT & Nombre de patients Nécessaires à Traiter \\
\hline PID & $\begin{array}{l}\text { Pain Intensity Difference (différence d'intensité de douleur rapportée pour } \\
\text { un intervalle de temps donné) }\end{array}$ \\
\hline SPID & $\begin{array}{l}\text { Sum of Pain Intensity Difference (somme pondérée par le temps des scores } \\
\text { d'intensité de douleur) }\end{array}$ \\
\hline TOTPAR & $\begin{array}{l}\text { TOTal Pain Relief (somme pondérée par le temps des scores de } \\
\text { soulagement de douleur) }\end{array}$ \\
\hline
\end{tabular}




\section{Introduction}

Le paracétamol («acetaminophen » aux Etats-Unis) est un antipyrétique et un antalgique de palier 1, largement prescrit et utilisé en automédication à travers le monde. En France, un rapport de l'Agence Nationale de Sécurité du Médicament et des produits de santé (ANSM) évaluait le paracétamol comme le principe actif le plus vendu en ville en $2013^{[1]}$. Malgré cela, son mécanisme d'action périphérique et central reste encore imparfaitement connu. Au niveau biochimique, le paracétamol agirait comme substrat des deux isoenzymes COX-1 et COX-2 pour inhiber la synthèse de prostaglandines. Son absence d'activité anti-inflammatoire et son apparente sélectivité pour COX-2 s'expliqueraient par la nécessité d'un contexte inflammatoire spécifique (faibles taux d'acide arachidonique et de peroxydes) pour pouvoir agir ${ }^{[2]}$. Des études récentes sur volontaires sains suggèrent une action complexe du paracétamol au niveau spinal et supraspinal, avec implication de récepteurs sérotoninergiques, opioïdergiques, vanilloïdes, cannabinoïdes, et de canaux calciques ${ }^{[3]}$. En pratique clinique, l'effet antalgique d'une prise orale unique de paracétamol versus placebo a été démontrée par des méta-analyses Cochrane pour 4 situations de douleur aiguë : les douleurs post-opératoires, les douleurs du post-partum, les céphalées, et les douleurs dentaires après extraction de dents de sagesse ${ }^{[4-7]}$. Le bénéfice de doses répétées de paracétamol est moins clair. Bien que les recommandations européennes préconisent l'utilisation du paracétamol en première intention pour les lombalgies aiguës communes $^{[8]}$, deux récentes méta-analyses n'ont pas pu démontrer le bénéfice d'un traitement prolongé par paracétamol dans ce contexte ${ }^{[9,10]}$.

Parce que le paracétamol est un antalgique largement utilisé en soins primaires, évaluer son efficacité dans ce contexte est un enjeu médical et pharmacologique primordial et évident. L'extrapolation de ce qui a été démontré dans quelques contextes cliniques à l'ensemble des situations de douleur aiguë est discutable. Nous proposons une méta-analyse des essais 
cliniques randomisés (ECR) pour évaluer l'effet antalgique d'une prise orale unique de paracétamol versus placebo chez l'adulte en soins primaires.

\section{Méthodes}

2.1.Identification et sélection des études

Les bases de données MEDLINE (via Pubmed), CENTRAL, et EMBASE (via OvidSP) ont été consultées le 4 février 2015 (équations de recherche disponibles sur demande). Tous les ECR en groupe parallèle et double aveugle, évaluant l'effet antalgique d'une prise orale unique de paracétamol versus placebo dans les 6 heures (h) suivant la prise du médicament chez l'adulte, ont été inclus. Les études évaluant un traitement préemptif (i.e., délivré avant que la douleur ne soit ressentie) ou donné chez des volontaires sains ont été exclues, de même que les essais en cross-over. Aucune restriction de langue n'a été appliquée. Pour l'analyse quantitative, seules les études réalisées en soins primaires ont été considérées. La sélection des études d'après le titre et le résumé, puis sur texte intégral, a été effectuée par un seul auteur (MG).

2.2. Critères de jugement considérés pour la méta-analyse

Compte tenu de l'absence de standardisation des résultats rapportés par les essais cliniques sur les antalgiques (possibilité de mesurer l'intensité et/ou le soulagement de la douleur, à des temps variables et en utilisant des échelles de mesure différentes d'une étude à l'autre), plusieurs critères de jugement ont été considérés pour l'analyse quantitative :

- La différence d'intensité de la douleur à $2 \mathrm{~h}$, critère de jugement fréquemment rapporté par les ECR et où le pic d'effet du paracétamol oral peut être observé $\left(\mathrm{PID}_{2 \mathrm{~h}}\right)^{[11]}$;

- Les sommes pondérées par le temps des scores d'intensité de douleur (SPID) et des scores de soulagement de la douleur (TOTPAR) rapportées par les patients sur $4 \mathrm{~h}$ ou $6 \mathrm{~h}$ $\left(\mathrm{SPID}_{4 \mathrm{~h}}, \mathrm{SPID}_{6 \mathrm{~h}}, \mathrm{TOTPAR}_{4 \mathrm{~h}}\right.$, et TOTPAR $\left.6 \mathrm{~h}\right)$; 
- Le nombre de patients soulagés d'au moins 50\% de leur douleur au cours des $4 \mathrm{~h}$ ou des 6h de suivi, estimés d'après les SPID ou les TOTPAR par les équations de Moore et $\mathrm{al}^{[12-14]}$.

Les résultats des critères de jugements continus ont été exprimés en différence de moyenne standardisée (DMS) avec un intervalle de confiance à 95\% (IC95\%). Pour les critères binaires, après réalisation d'un modèle d'effet (i.e., régression linéaire simple utilisant les données de chaque étude pour décrire le risque sous paracétamol en fonction du risque sous placebo), nous avons finalement choisi d'exprimer les résultats en différence de risque (DR) avec un intervalle de confiance à 95\% (modèle d'effet non présenté dans cet article). Cet indice présentait moins d'hétérogénéité entre études que le risque relatif et l'odds ratio.

\section{Résultats}

\subsection{Description des études}

L’interrogation des 3 bases de données a identifié 5802 références. Après sélection sur titre et résumé, 194 rapports d'études répondaient à nos critères d'inclusion. Les contextes cliniques évalués par au moins 2 ECR étaient les suivants : douleurs post-opératoires ; douleurs dentaires (quasi exclusivement post-chirurgicales); céphalées ; douleurs du post-partum ; et maux de gorge. Le paracétamol oral versus placebo était également évalué dans l'arthrose, l'arthrite rhumatoïde, les douleurs abdominales, les dysménorrhées et les lombalgies communes, mais uniquement à des doses répétées et avec une évaluation de la douleur au mieux quotidienne pour 14/19 références. Dans les 5 autres cas, le paracétamol était utilisé comme traitement de secours, donné par voie intraveineuse, ou n'était pas comparé à un placebo. Vingtdeux références restent en attente de classification, faute de document disponible.

Après lecture sur texte intégral et analyse de la bibliographie de chaque article, nous avons retrouvé $21 \mathrm{ECR}$ de soins primaires ou concernant une pathologie de soins primaires, 
ayant évalué l'effet antalgique d'une prise orale de paracétamol versus placebo chez l'adulte. Six ECR ont dû être exclus, faute de données exploitables pour l'analyse quantitative. Les 15 ECR restants concernaient les maux de gorge (5 ECR ; 842 patients) et les céphalées (12 ECR ; 3433 patients), 2 ECR évaluant l'effet du paracétamol dans ces 2 indications simultanément (389 patients). Aucune étude n'était exploitable pour les lombalgies. Les doses de paracétamol étudiées étaient de $500 \mathrm{mg}$ (2 études), $650 \mathrm{mg}$ (1 étude), et $1000 \mathrm{mg}$ (14 études). Le diagramme de sélection des articles est présenté en figure 1. Les références incluses dans cette méta-analyse sont disponibles sur demande.

\subsection{Evaluation de l'effet antalgique du paracétamol en soins primaires}

Quel que soit le critère de jugement considéré, l'effet antalgique du paracétamol était significativement supérieur à celui du placebo. Concernant l'estimation du nombre de patients soulagés à 4 h ou à 6 d'après les scores d'intensités ou de soulagement de douleur, 6 à 12 études (1736 à 3416 patients) étaient comparables pour ces 4 critères de jugement. La différence de risque allait de $0.19(\mathrm{IC} 95 \%=0.11-0.27)$ à $0.23(\mathrm{IC} 95 \%=0.17-0.30)(\mathrm{p}<0.00001$ pour chaque comparaison), soit un nombre de patients nécessaires à traiter (NNT) allant de 4 à 5 . Concernant les critères de jugement continus, la plus grande différence observée concernait la $\mathrm{PID}_{2 \mathrm{~h}}$, avec une DMS à 0.92 (IC95\%=0.30-1.54; p=0.004; 4 études, 917 patients). Pour le SPID $4 \mathrm{~h}$, SPID $_{6 \mathrm{~h}}$, TOTPAR $_{4 \mathrm{~h}}$ et TOTPAR $_{6 \mathrm{~h}}$, les DMS étaient respectivement de 0.51 (IC95\%=0.04-0.98; $\mathrm{p}=0.03 ; 4$ études, 754 patients), de 0.73 (IC95\%=0.30-1.15; p=0.0009; 5 études, 1419 patients), de 1.02 (IC95\%=0.20-0.91; p=0.002; 4 études, 787 patients) et de 0.81 (IC95\%=0.36-1.26; p=0.0004; 5 études, 1562 patients). Il existait une forte hétérogénéité entre les études pour les 9 critères de jugement ( $\mathrm{I}^{2}$ de 57 à $95 \%$ ), malgré le choix d'un modèle à effets aléatoires. 
L'influence de la dose de traitement et du contexte clinique a été étudiée par 2 analyses en sous-groupe. Un seul critère (le nombre de patients soulagés à 4h d'après les scores d'intensité de douleur) a permis la comparaison du paracétamol $500 \mathrm{mg}$ (2 études, 279 patients) versus 1000 mg (12 études, 3137 patients). Dans cette comparaison, seul le paracétamol 1000 mg avait un effet significativement supérieur au placebo $\left(\mathrm{DR}_{\mathrm{P} 10000}=0.20 ;\right.$ IC95\%=0.12-0.28 ; $\mathrm{p}<0.00001$; versus $\left.\mathrm{DR}_{\mathrm{P} 500}=0.15 ; \mathrm{IC} 95 \%=-0.02-0.31 ; \mathrm{p}=0.08\right)$. Concernant l'influence du contexte clinique, la comparaison a pu être effectuée pour les 4 critères binaires ainsi que pour le TOTPAR 6 h $(3$ à 10 ECR sur les céphalées, soit 1240 à 3064 patients par comparaison ; 2 études sur les maux de gorge soit 199 à 322 patients par comparaison). L'effet antalgique du paracétamol dans les céphalées était supérieur au placebo pour les 5 critères de jugement. Pour les maux de gorge, la différence d'effet n'était pas significative, du fait d'un intervalle de confiance très large (faible nombre de patients comparés pour cette indication). L'analyse en sous-groupe pour le critère de jugement « nombre de patients soulagés d'au moins $50 \%$ de leur douleur à 4h d'après les intensités de douleur rapportées » est présentée en figure 2.

\section{Discussion}

Si le paracétamol est le médicament le plus vendu en médecine de ville en France ${ }^{[1]}$, son effet antalgique n'a été évalué que dans 2 contextes cliniques de soins primaires : les maux de gorge et les céphalées. Ces 2 indications représenteraient respectivement $4.7 \%$ et $3.3 \%$ des motifs de consultation de médecine générale d'après l'étude ECOGEN (L. Letrilliart, communication personnelle, 6 février 2017). D’autres indications fortement prévalentes en soins primaires, telles que les lombalgies $(7,3 \%$ des consultations de médecine générale selon l'étude ECOGEN $\left.{ }^{[15]}\right)$, sont à ce jour inexplorées.

L'absence de texte intégral disponible pour 22 références est une limite à ce travail. Cependant, les éléments apportés par le titre et le résumé (lorsqu'il est présent) laissent penser 
que ces références ne donnent pas de données exploitables pour les 2 situations cliniques évaluées dans cette méta-analyse ni pour les lombalgies, et ne devraient pas modifier les résultats actuels. L'autre limite vient du fait que la sélection des articles et l'analyse des données n’ont été réalisées que par un seul auteur. La mise à jour et la répétition de ce travail par 2 auteurs indépendants est en cours.

Comme rapporté dans d'autres méta-analyses ${ }^{[4-7]}$, l'effet d'une dose de paracétamol oral était significativement supérieur au placebo dans notre étude, quel que soit le critère de jugement considéré. L'analyse de sensibilité pourrait suggérer qu'il existe une influence du contexte clinique sur l'effet du traitement, mais le petit nombre de patients dans le groupe « maux de gorge » gêne l'interprétation de ces résultats. Afin de confirmer ou non cette hypothèse, et d'étayer les indications pour lesquelles l'effet antalgique du paracétamol a été validé, nous proposons de réaliser un ECR de soins primaires comparant l'effet d'une prise orale de paracétamol versus placebo dans deux situations cliniques de forte prévalence : les maux de gorge et les lombalgies aiguës.

Conflits d'intérêts : aucun.

\section{Références :}

[1]. ANSM. Analyse des ventes de médicaments en France en 2013. Juin 2014.

http://ansm.sante.fr/var/ansm_site/storage/original/application/3df7b99f8f4c9ee634a6a9b094624341.pdf, consulté le 20 janvier 2017 (36 pages).

[2]. Graham GG, Davies MJ, Day RO, et al. The modern pharmacology of paracetamol: therapeutic actions, mechanism of action, metabolism, toxicity and recent pharmacological findings. Inflammopharmacology 2013; 21(3): 201-32.

[3]. Pickering G, Kastler A, Macian N, et al. The brain signature of paracetamol in healthy volunteers: a doubleblind randomized trial. Drug Des Devel Ther. 2015 Jul 23; 9: 3853-62. 
[4]. Toms L, McQuay HJ, Derry S, et al. Single dose oral paracetamol (acetaminophen) for postoperative pain in adults. Cochrane Database Syst Rev 2008; (4).

[5]. Chou D, Abalos E, Gyte GML, et al. Paracetamol/acetaminophen (single administration) for perineal pain in the early postpartum period. Cochrane Database Syst Rev 2013; (1).

[6]. Derry S, Moore RA. Paracetamol (acetaminophen) with or without an antiemetic for acute migraine headaches in adults. Cochrane Database Syst Rev 2013; (4).

[7]. Weil K, Hooper L, Afzal Z, et al. Paracetamol for pain relief after surgical removal of lower wisdom teeth. Cochrane Database Syst Rev 2007; (3).

[8]. Van Tulder M, Becker A, Bekkering T, et al. Chapter 3. European guidelines for the management of acute nonspecific low back pain in primary care. Eur Spine J 2006; 15 (Suppl. 2): S169-91.

[9]. Davies RA, Maher CG, Hancock MJ. A systematic review of paracetamol for non-specific low back pain. Eur Spine J 2008; 17(11): 1423-30.

[10]. Machado GC, Maher CG, Ferreira PH, et al. Efficacy and safety of paracetamol for spinal pain and osteoarthritis: systematic review and meta-analysis of randomised placebo controlled trials. BMJ 2015; 350: h1225.

[11]. Moller PL, Sindet-Pedersen S, Petersen CT, et al. Onset of acetaminophen analgesia: comparison of oral and intravenous routes after third molar surgery. Br J Anaesth 2005; 94(5): 642-8.

[12]. Moore A, McQuay H, Gavaghan D. Deriving dichotomous outcome measures from continuous data in randomised controlled trials of analgesics. Pain 1996; 66(2-3): 229-37.

[13]. Moore A, McQuay H, Gavaghan D. Deriving dichotomous outcome measures from continuous data in randomised controlled trials of analgesics: verification from independent data. Pain 1997; 69(1-2): 127-30.

[14]. Moore A, Moore O, McQuay H, Gavaghan D. Deriving dichotomous outcome measures from continuous data in randomised controlled trials of analgesics: use of pain intensity and visual analogue scales. Pain 1997; 69(3): 311-5.

[15]. Pernollet E, Ramond-Roquin A, Fouquet N, et al. La lombalgie chez les adultes consultant en médecine générale : fréquence, caractéristiques sociodémographiques et résultats de consultation associés. Exercer 2014 ; $114: 170-2$ 
Fig. 1. Diagramme de sélection des études incluses dans la méta-analyse.

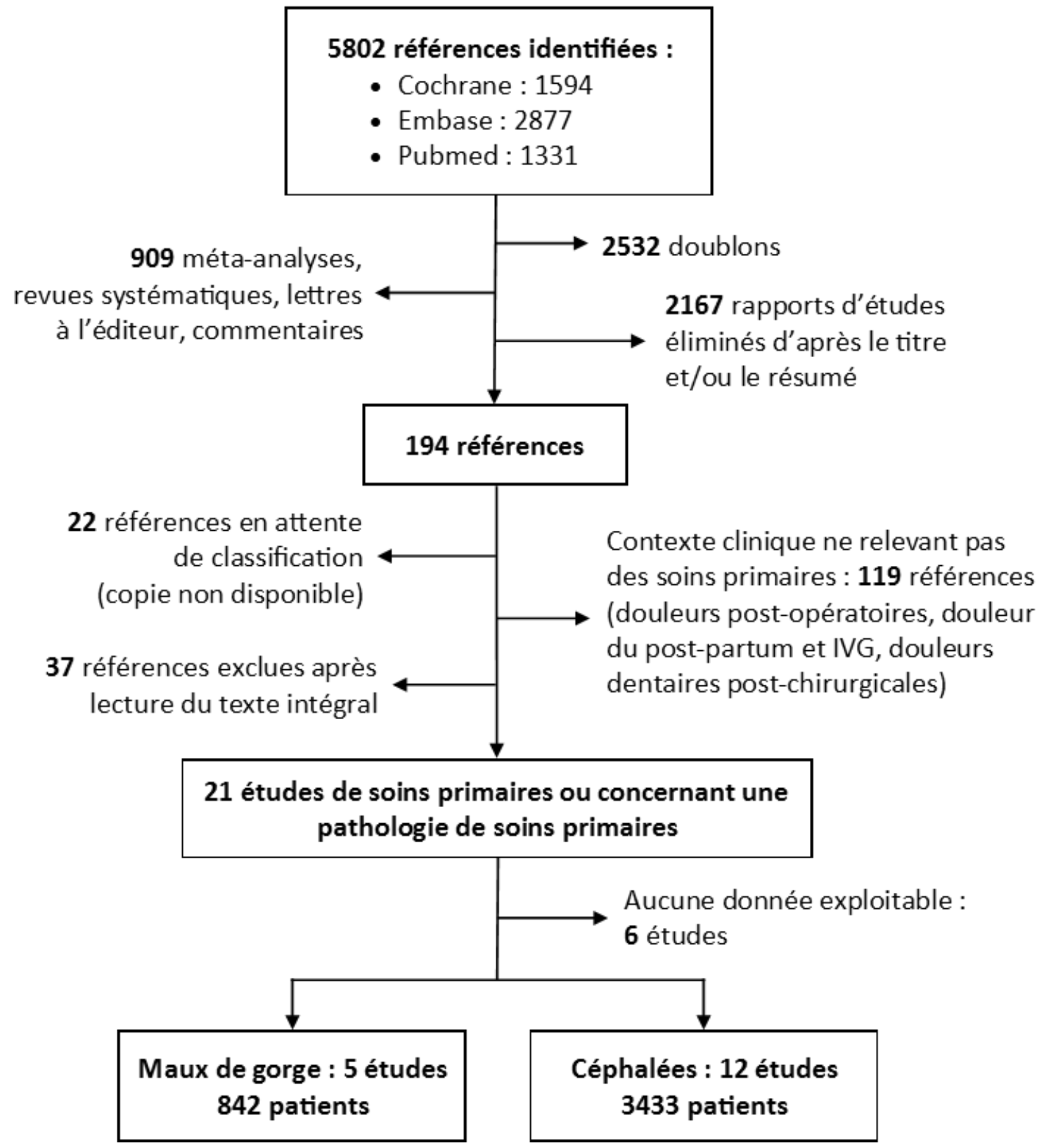


Fig. 2. Analyse en sous-groupe en fonction du contexte clinique évalué, pour le critère de jugement «nombre de patients soulagés d'au moins $50 \%$ de leur douleur à 4 h d'après les intensités de douleur rapportée »

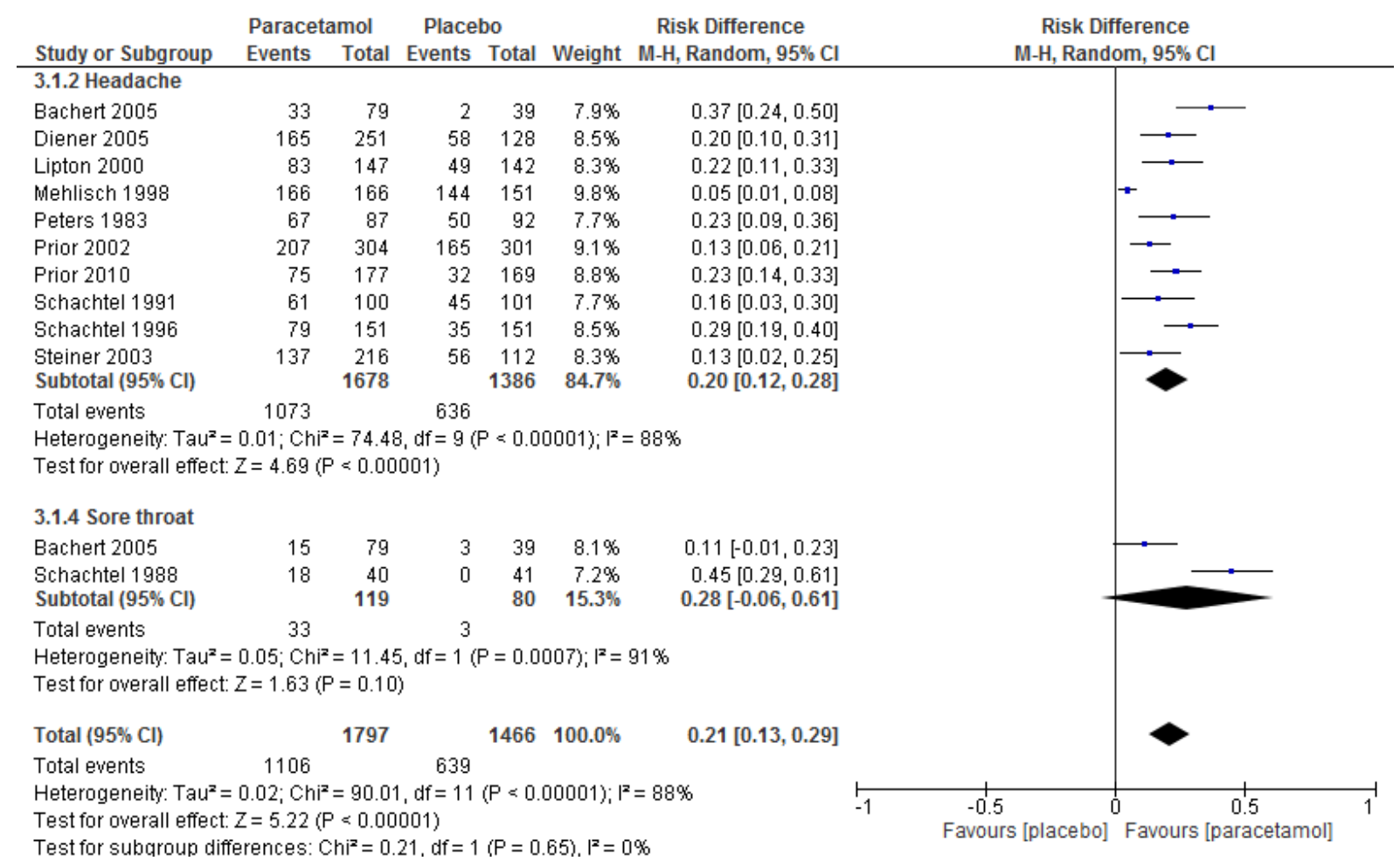

